\title{
Introduction : énigme, prière, identité
}

Cette étude se propose d'examiner l'œuvre d'Esther Tellermann (1947-), poète majeur dont les recueils explorent depuis une quarantaine d'années l'intime du monde, ses textures et ses contours, ses terres insituables semblant s'entrecroiser, le rêve et le mythe faits tremplin pour une traversée toujours renouvelée de l'Histoire et du deuil. Il s'agit d'une parole à la fois ample, souple, ouverte, fluide et mesurée, offerte à l'Autre pour s'entretenir avec lui. Structurés en longues séries de chants, ce sont des poèmes qui évitent toute résolution, préférant moduler un récit énigmatique sans cesse repris. Les enjeux en sont considérables, car le « lyrisme décentré » de Tellermann ${ }^{1}$ veut agir peu à peu sur le lecteur, le mettre au centre du dire en l'obligeant à rester attentif à la venue en présence des êtres et des choses, à la réalité sensible autant qu'aux disparus qui nous restent proches.

Nous mettrons en relief la période 1999-2019, de Guerre extrême à Unversant l'autre. Les recueils de cette période forment un tout extrêmement cohérent, dès que l'on accepte de rester patient, d'accueillir les archipels de mots et de motifs, de suivre avec le regard et l'âme un réel en pleine métamorphose. La douleur n'en sera pas absente, mais elle sera adoucie par le mystère attirant du réel, le flux et le reflux de ses plis offerts à notre regard, l'intonation et les cadences par lesquelles ce mouvement nous atteint. Ellipses, incises, polysémie, refrains, reprises : nous chercherons à comprendre ce que fait Tellermann en acceptant de nous acheminer avec quelqu'un dont la parole nous permet d'« advenir », ${ }^{2}$ de nous plonger dans le langage pour entrer en relation avec soi et l'Autre, l'Histoire et le changement, l'ici et l'ailleurs. Nous verrons comment la force que nous donnent les mots se dégage non seulement d'énoncés et de textes isolés, mais de textes conçus comme suites qui s'enchaînent, tant à l'intérieur de chaque livre que de recueil en recueil.

1 Alain Cressan, « Esther Tellermann: Avant la règle/Un point fixe », CCP : cahier critique de poésie 29.2 (déc. 2014), <cahiercritiquedepoesie.fr>. Cressan parle aussi d'un «je / nous abstrait, autre, qui se situe plus dans l'adresse au tu / vous, que dans l'expression d'un moi » (l'auteur souligne).

2 UV 69 : « en mots simples advenir ». Une autre image qui semblerait aller dans ce même sens (arriver accidentellement, par hasard, par le biais du langage mais sans que l'on s'y attende) est celle chez Philippe Jaccottet, et qui fait penser aussi à André du Bouchet, d'un « dépliement de fraîcheur » aux niveaux des mots et du réel. Jaccottet souhaite décrire « comment [les choses] s'ouvrent, et comment nous entrons dedans ». Voir Philippe Jaccottet, Paysages avec figures absentes, Paris : Gallimard, 1976, p. 68 et p. 17 .

(C) AARON PREVOTS, 2022 | DOI:10.1163/9789004499683_002 
Au cœur de nos lectures attentives seront les poèmes de Paul Celan (19201970), écrivain de langue allemande qui a profondément souffert à cause de la Shoah et de l'antisémitisme. Celan incarne l'exil et la différence, notions qui fascinaient Tellermann lors de voyages à l'étranger dont le récit énigmatique sous-tend par moments ses poèmes. Chassé de son pays, la Bucovine, où l'on parlait le roumain, s'étant débarrassé de son vrai nom Antschel pour écrire sous un pseudonyme, s'étant mis à publier en allemand, la langue préférée de ses parents avant leur mort violente et surtout de sa mère, qui le choyait, respecté par ses pairs à Paris mais jamais tout à fait à l'aise à cause de fausses accusations de plagiat, Celan se donnait pour objectif la refonte de structures poétiques et ainsi de tout ce qui nous aide à être - à exister, imaginer, partager, rêver, s'entendre. Il voulait que les juifs se sentent chez eux dans la langue, que leurs racines ne soient pas, suite aux génocides, uniquement dans le ciel. Il priait pour eux, pour leur redonner l'identité qui leur avait été volée. Il devait le faire de manière énigmatique, s'appuyant sur le langage pour les recoudre dans la trame de l'humanité, contre les discours contemporains ainsi que celui des oppresseurs. ${ }^{3}$

Tellermann, pour sa part, après Première apparition avec épaisseur (1986), Trois plans inhumains (1989), Distance de fuite (1993) et Pangéia (1996), s'est intéressée de plus en plus à Celan, comme technicien capable de mettre en place en langue allemande des outils inattendus d'une part, et, de l'autre, comme bâtisseur d'images concernant des mots renouvelés à porter aux lèvres, à offrir à autrui, à retirer des cendres pour qu'ils fleurissent, luisent, témoignent, nous remplissent les mains, retrouvent le sens. ${ }^{4}$ Le style de ces quatre premiers recueils de Tellermann a son caractère propre. Cependant, de temps à autre, le lexique, le dépouillement et l'éparpillement de vers brefs peuvent faire penser à Celan, par exemple là où les mots sont « comme neige entendue / flocons »,

3 Quant à la vie de Celan et les liens entre lui et le destin de tout un peuple, voir « Présentation : Paul Celan, 1920-1970 », Poèmes, trad. et éd. John E. Jackson, Paris : Corti, 2007, p. 9-84. Voir aussi John Felstiner, Paul Celan : Poet, Survivor, Jew, New Haven : Yale UP, 2001. Jackson détaille « le développement d'une bourgeoisie juive germanophone » à Czernowitz, ville natale de Celan, et « l'amour de l'allemand » que la mère du poète valorisait par rapport au yiddish ou à l'hébreu (p. 12 et p. 14).

4 Pour des remarques de la part de Tellermann sur l'écriture de Celan, voir Aaron Prevots, «Entretien avec Esther Tellermann », French Review 92.4 (May 2019), p. 101-13 (108-10). Vis-àvis de sa fascination pour Celan en tant que témoin de la Shoah, de l'exil et de la poésie réparatrice, voir notamment Esther Tellermann, « Cristal exact », Paul Celan, Europe 1049-1050 (sept.-oct. 2016), p. 43-48 (45), où elle s'adresse à son aîné : « Il me fallut vous répondre "en poème", comme pour rendre compte de ces territoires toujours autres, pays perdus, ni arrachés, ni à recouvrer, mais depuis toujours perdus, faits poésie, entremêlant le ciel et l'eau, la lune et le gel, où nous avons à nous tenir debout. » 
là où de la terre « jaillit la brume bleue » $(P A 86-87)$. La « prière » y est « balancement» (DF 68 ; cf. $P A$ 19) - du corps, du regard, des strophes. La versification fait écho au « vertige / élargi » $(D F 44)$. On peut constater l'envie de transformer «la plainte / en polyphonie » ( $D F$ 4O), même si le ton reste le plus souvent sobre, les émotions très contenues. Lallemand - «stimmen », « augen » (PG 100) - et de brèves citations de Celan surgissent comme une sorte de «poussière dans la main » (PG 103), comme des fragments supplémentaires pour mettre en perspective toutes sortes d'histoires humaines, celles d'autres ères et celles, chagrinées et déchirantes, qu'a dépeintes Celan en écrivant sa fameuse « Todesfuge » sur la mort, ce « songe venu d'Allemagne » $(D F 72) .{ }^{5}$

En outre, l'Est attire forcément Tellermann. Quand on pense à son œuvre dans son ensemble, il paraît normal que son intérêt pour Celan aille croissant. On pourrait signaler ses origines juives polonaises, ${ }^{6}$ mais c'est de toute une tradition littéraire qu'il s'agit pour elle en lisant Celan, loin des débats français sur le lyrisme, la littéralité ou toute autre voie que la critique aime à voir les poètes suivre. Celan est un écrivain qui peut jouer sur la moindre syllabe, colorer des horizons affectifs même avec un lexique restreint, mettre en musique ce qui le ronge, crier en baissant la voix. Il recueille les plaintes des morts en gardant en mémoire les diasporas auxquelles ils avaient appartenu, ainsi que leurs traditions religieuses et philosophiques. Sa grande culture littéraire et linguistique, sa maîtrise formelle novatrice, sa pensée audacieuse et inquiète, et son envie de témoigner font de lui un poète incontournable de l'après-guerre, ainsi qu'un maillon fort dans la chaîne des générations.

Toutes ces dimensions font partie des palimpsestes que l'on peut cerner dans les poèmes de la deuxième période de Tellermann. ${ }^{7}$ Celan n'y est pas mentionné, mais de nombreux aspects s'inspirent de son dire ou lui sont empruntés. Dans les recueils de 1999-2019, tout un appareil de mots et d'images semés ici et là dans le récit font croire que c'est lui l'Autre, voire le bien-aimé avec qui

5 Cf. «Todesfuge » / « Fugue de mort », Paul Celan, Pavot et Mémoire, trad. Valérie Briet, Paris : Christian Bourgois, 1987, p. 83-89. Dans « Poésie », Journal français de psychiatrie 27 (2006/4), p. 5on5, <cairn.info>, Tellermann dira que Celan est « [1]e plus grand poète de la seconde moitié du XXe siècle ».

6 Aaron Prevots, «Entretien avec Esther Tellermann », op. cit., p. 108 ; cf. Patrick Née, « Poème et identité : entretien avec Esther Tellermann », « Dossier Esther Tellermann » (éd. Jean-Baptiste Para), Éric Chevillard.Jean-Louis Giovannoni. Esther Tellermann, Europe 1026 (oct. 2014), p. $146-55$ (148).

7 Nous nous permettons d'emprunter à Yves Di Manno et Isabelle Garron cette notion d'une « seconde période», en l'adaptant. Ils la datent de 1999 à 2010, et nous l'étendons à 2019. Voir Yves Di Manno et Isabelle Garron, éd., Un nouveau monde : poésies en France 1960-2010. Un passage anthologique, Paris, Flammarion, 2017, p. 1049-61 (1051). 
coucher, nager, parler, regarder. Néanmoins, comme cette étude le montrera, ce sont des palimpsestes au pluriel que l'on peut remarquer, par exemple la présence en sourdine de Friedrich Hölderlin (1770-1843), Gérard de Nerval (18081855), Georg Trakl (1887-1914), Saint-John Perse (1887-1975), Pierre Reverdy (1889-1960), Ossip Mandelstam (1891-1938) et André du Bouchet (1924-20o1), pour ne citer que quelques noms liés directement ou implicitement à l'œuvre de Tellermann. Ce sont tous des êtres en quête de vérités provisoires et qui se laissent porter par les écumes du désir. Ils font face poétiquement aux écueils des discours quotidiens, font chanter un sujet emporté par le désir et les signes, ${ }^{8}$ trouvent dans le réel du poème la matière qui les fait vivre.

Comme on le verra, le déploiement de la parole de Guerre extrême à Un versant l'autre présente un mélange de fluidité et de questionnement qui appartiennent à Tellermann entièrement. Son écriture autoréflexive croise d'autres voix, mais aura toujours ses propres couleurs, ses propres qualités sonores, son imaginaire qui rend la terre « musicale / fruitée teintée / suave $[\ldots]$ bigarrée comme un cri » (ER 73). S'y opère un dépassement de l'amertume, des reflets de ce que Martine Broda appelle, après Lacan, un « désir qui ne désire rien, sinon perdurer, désir de désirer $\gg .^{9}$ Le Je lyrique tient compte souvent d'un « autre versant » où « tout serait » $(P G$ 7), ou bien de variantes de ce leitmotiv, par exemple l'autre bord, côté ou rive. Les départs reprennent, sans qu'on arrive jamais à un lieu déterminé. Enfin, il faudrait noter que les quelques auteurs auxquels nous réfléchirons ne sont qu'une porte qui ouvre sur ce que fait Tellermann. Si nous mettons l'accent sur les recueils de 1999-2019, c'est parce que la parole de Tellermann s'y déploie pleinement, trouve tout son éclat en se concentrant sur le «chant des morts enfoui sous la parole des vivants », en méditant celui-ci « souveraine[ment], tournée vers quels dieux absents? » ( $E R 4^{\mathrm{e}}$ de couv., l'auteur souligne). C'est dans ces zones d'ombre que l'épopée prend son envol.

8 Martine Broda, «Lyrisme et célébration : l'épiphanie de la Chose », Littérature 104 (1996), p. 89-100. Cf. Tellermann à propos de Lacan et la poésie comme reflet du travail de l'inconscient dans la vie de tous : «J'ai rassemblé récemment un ensemble de textes critiques divers sous cette formule empruntée à Lacan: "Nous ne sommes pas pouâtassez", où nous entendons "poète assez" ou "poétassier", que j'ai reprise ainsi plus classiquement par ce titre : "Nous ne sommes jamais assez poète" » (Esther Tellermann, « Esther Tellermann : le travail du poète », École pratique des hautes études en psychopathologies, 21 nov. 2012, p. 4, <ephep .com>). Voir aussi $N N$.

9 Ibid., p. 92. Voir aussi Aaron Prevots, «Entretien avec Esther Tellermann », op. cit., p. 109 : «Dans quel espace se mouvoir afin que la différence soit possible? La "personne" celanienne rejoint l'Autre lacanien, me semble-t-il, cette instance tierce, symbolique, nécessaire au désir. Cette instance tierce sépare du Un qui souvent fait la passion humaine. J'ai modestement trouvé dans le poème cet espace, cette "Terre exacte" ». 
Quelques mots d'ordre bio-bibliographique s'imposent. Agrégée de lettres, Tellermann exerce actuellement la psychanalyse et a participé régulièrement à diverses revues, par exemple Europe, le Bulletin de l'Association lacanienne internationale et La Célibataire. Elle fait partie des comités de rédaction du Journal français de psychiatrie et de La Revue lacanienne. Elle a publié une vingtaine de livres de poésie, ainsi que deux récits, Une odeur humaine (2006) et Première version du monde (2018). Nous ne sommes jamais assez poète (2014) rassemble une trentaine de ses textes critiques. On lui a décerné le grand prix de l'Académie Française pour Première apparition avec épaisseur en 1986, le prix François Coppée de l'Académie Française pour Guerre extrême en $2000^{10}$ et le prix Max Jacob pour Sous votre nom en 2016. ${ }^{11}$ On lui a consacré le numéro 39 de la revue $N U(e)$ en 2008, un dossier pour le numéro 1026 de la revue Europe en 2014 et un dossier pour le numéro 56 de L'Étrangère en $2022 .{ }^{12}$ Est apparu en 2020 son recueil Corps rassemblé, sur les incandescences du corps féminin, écrit suite à plusieurs visites de l'atelier du peintre Claude Garache. Une notice dans une anthologie récente soulève des faits concernant sa vie professionnelle : l'intégration de l'École normale supérieure en 1968, une carrière d'enseignante de lettres dans le secondaire, le travail clinique qu'elle mène, une « quête des origines » d'ordre poétique. ${ }^{13}$

Cette quête d'ordre poétique va de pair avec son dynamisme professionnel, par exemple sa direction de colloques sur Antonin Artaud (1896-1948), Michel Deguy (1930-), Bernard Noël (1930-2021) ou François Rouan (1943-) (LP, MD, $B N, F R)$. Ne perdons pas de vue le fait que, selon Tellermann, le moi du poète s'efface derrière « le langage, le trésor des signifiants » : « l'œuvre littéraire ne rév[èle] aucune personnalité de l'auteur, mais cet Autre en lui, grand Autre, où l'écrivain voit le plus souvent sa vocation ».14 À partir de 1999, ce trésor des

\footnotetext{
$10 \quad$ Voir aussi $M G$.

11 Cf. Esther Tellermann, « Pour saluer Max Jacob », Les Cahiers Max Jacob 15/16, Pau : Les Amis de Max Jacob, 2015, p. 97-101, <cahiersmaxjacob.org $>$.

12 Laurent Fourcaut, éd., Esther Tellermann, NU(e) 39, 2008 ; Jean-Baptiste Para, éd., « Dossier Esther Tellermann », Éric Chevillard. Jean-Louis Giovannoni. Esther Tellermann, Europe 1026 (oct. 2014), p. 140-91 ; François Rannou, éd., « Dossier Esther Tellermann », L'Étrangère 56 (printemps 2022).

Yves Di Manno et Isabelle Garron, éd., Un nouveau monde : poésies en France 1960-2010, op. cit., p. 1049. Quant aux premières étapes de la vie professionnelle de Tellermann, voir Anne Malaprade, «Entretien avec Esther Tellermann », Le Nouveau Recueil 70 (mars-mai 2004), p. 162-73 (162-64). Sur les enjeux de l'enseignement vis-à-vis de ses trente-neuf ans « dans un collège de la banlieue nord de Paris puis en lycée technique », par exemple son désir d'« échappe[r] aux discours courants », voir « Enseigner aujourd'hui ? », Journal français de psychiatrie 31 (2007/4), p. 4-5, <cairn.info>.

Esther Tellermann, « Esther Tellermann : le travail du poète », op. cit., p. 5.
} 
signifiants comprend « un peuple, une communauté et des singularités présentées par les pronoms (nous, vous, je, tu, ils) »; il est question « de lieux habités », de liens à nos semblables, « sans que jamais ne soit fixée la nature du rapport entre les innomés ». ${ }^{15}$ En somme, quoi que l'on fasse, on est rarement dans le biographique, toujours du côté de signifiants, de "silences neufs », 16 de sens pluriels et équivoques par rapport au poète et à ses destinataires ou interlocuteurs, au sein d'une œuvre, dès le début des années 2000, déjà « toute de maturité ». ${ }^{17}$

Qu'est-ce qui a donc lieu dans les poèmes narratifs que nous aborderons? Quels gestes ou envies rendent le jeu des signifiants apaisant, intersubjectif, sensoriel, nécessaire ? Comment les enjeux deviennent-ils communautaires? Voici la liste des recueils auxquels nous nous intéresserons, puis des verbes qui annoncent les chapitres et leurs problématiques : Guerre extrême (1999), Encre plus rouge (2003), Terre exacte (2007), Contre l'épisode (2011), Sous votre nom (2015), Le Troisième (2013), Éternité à coudre (2016), Avant la règle (2014), Carnets à bruire (2014) et Un versant l'autre (2019); accompagner, accueillir, adoucir, aviver, bruire, commémorer, consoler, déployer, étreindre, nommer, ouvrir, prier, réconcilier, reconstruire, recueillir, réparer, repriser, restituer, rêver, se souvenir, s'innocenter, soulager, s'ouvrir, traverser, veiller. Bien qu'elliptiques, les poèmes de Tellermann entrent dans les énigmes pour ces raisons précises, sertissant le dire et mettant en lumière des actes de parole. Notre méthode sera d'aborder chaque livre ou groupe de livres en cherchant son noyau dur. Nous décrirons ce qui s'y trame et cernerons les objectifs de l'auteur suggérés par ses mots, images et structures, y compris ses emprunts à Celan, dans lesquels s'ancrent de plus en plus son dire pendant ces deux décennies. Seront mis en avant un réel mouvant aux contours variables, un questionnement et un dialogisme constants, une plénitude - du dire, du devenir - que l'accent même mis sur des secrets difficilement percés augmente.

Nous examinerons progressivement diverses sortes de dialogues poétologiques. Si par exemple Mallarmé commente le coup de dés qui donne lieu à l'espace textuel, ${ }^{18}$ Césaire le retour cathartique au pays natal, ${ }^{19} \mathrm{chez}$

\footnotetext{
15 Yves Di Manno et Isabelle Garron, éd., Un nouveau monde, op. cit., p. 1051.

16 Gérard Noiret, « Deux voix plus une : Esther Tellermann, Le Troisième », La Nouvelle Quinzaine litteraire 1111 (o1 sept. 2014), p. 15.

17 Jean-Marie Perret, «Esther Tellermann : Encre plus rouge », $C c P$ : cahier critique de poésie 7.1 (2003), p. 114.

18 Stéphane Mallarmé, Un coup de dés jamais n'abolira le hasard, in OEuvres complètes, éd. Henri Mondor et G. Jean-Aubry, Paris : Pléiade, 1945, p. 453-77.

19 Aimé Césaire, Cahier d'un retour au pays natal, in Anthologie poétique, éd. Roger Toumson, Paris : Imprimerie nationale, 1996, p. 37-83.
} 
Tellermann la rencontre avec l'œuvre celanienne est de même un repère charnière, emblématique, indissociable de son dire. C'est un lieu à partir duquel se développent l'imaginaire, le face-à-face avec l'abîme et les mots lancés qui vont se caresser ou s'entrechoquer. Le chapitre 1 entrera dans le vif du sujet en parlant des «psaumes détournés » (GE 107) de Guerre extrême, où Tellermann fait ressortir les peines de Celan et leur guerre commune avec l'indicible du passé, les trous de l'Histoire. Ce premier aperçu de l'intertextualité chez Tellermann examinera l'opacité et les zones d'ombre de ce recueil, mais montrera aussi que le dire poétique en tant que rituel peut apaiser, guérir, nous réconcilier avec la violence, nous mener vers des lieux affectifs où il est possible de mieux voir, respirer, communiquer. Si guerre il y a, elle nous apprend selon Celan à explorer en songe la mémoire collective, à nager à travers le sel des signes pour retrouver autrui. Auprès de ce passeur, Tellermann apprend à errer dans l'inconscient et le langage, à mieux retrouver le contact avec les ténèbres par le poème. Si les textes celaniens auxquels fait allusion Tellermann nous aident à suivre le fil du poème, il n'en reste pas moins vrai que la structure et le phrasé de Guerre extrême sont au cœur de la mémoire qui se construit. Notre lecture attentive comprendra l'étude des emprunts aux Psaumes et du cheminement spirituel auxquels ils s'associent.

Le chapitre 2 suivra la progression par laquelle Encre plus rouge chante les morts. À cet égard sera analysée la manière dont le devenir s'inscrit dans le déploiement du temps et de l'espace, ainsi que dans le développement des trois séquences narratives. Nous discuterons de l'inscription dans la trame du récit de mondes qui s'entrelacent, afin d'en montrer les dimensions humaine et mythique, notamment la présence de l'Antiquité, de mots profondément polysémiques et d'allusions qui ouvrent des horizons spirituels et affectifs. Nous verrons comment, au-delà de l'exotisme qui nous plonge dans un lieu mythique, attirant, coloré, minéral, le souffle ample - comme inépuisable - et les figures sonores renforcent la notion qu'un « signe mat et clair » (ER 188) peut venir à notre secours, à la façon d'une « sonate » (ER 218). Vis-à-vis de l'intertextualité, nous creuserons tout particulièrement sa manière de citer SaintJohn Perse, officiant souvent joyeux avec qui elle lève les yeux vers l'écume et l'éternel.

En effet, Tellermann ne traite pas que du deuil. Comme Reverdy ou Du Bouchet, Perse ou Supervielle (1884-1960), elle embrasse tout l'univers, la terre et le ciel, les choses et les êtres. Elle structure ses livres de telle manière que leur cadre laisse s'installer un flottement, un « balancement » (DF 68), un bercement. Il est important d'insister là-dessus, pour dire l'envergure de sa poétique à partir des années 2000 : la pensée souple, le jeu des pronoms et des vocatifs, les routes et ciels variés, nuancés. Une spécificité de son œuvre 
dans cette période est l'aspect généreusement tellurique, ${ }^{20}$ comme si les choses simples de la terre, présentées avec précision, concision et fréquence, laissaient percevoir l'ouvert, pouvaient être une source d'espoir et d'équilibre, d'ancrage et d'incandescence. Le chapitre 3 est donc consacré aux rites et à la rêverie dans Terre exacte. Nous contemplerons le tellurique comme tremplin vers la commémoration du passé récent, la compréhension d'un passé collectif mythique, le renouvellement de liens avec l'élémentaire et la récupération d'une plus grande stabilité au plan de l'inconscient. Nous explorerons le lexique et les motifs empruntés à Celan par lesquels Tellermann donne voix aux disparus, met en évidence les liens identitaires et générationnels que crée le langage et que parle la terre pour souligner le devenir, le sacré, le dialogue à instaurer avec le dehors.

Dans le quatrième chapitre, nous rendrons compte, par le biais de Celan, de la Shoah en tant que traumatisme collectif. Nous poserons des questions auxquelles Contre l'épisode apporte des réponses, parlant certes de ce traumatisme et de l'identité juive, mais aussi des gestes poétiques chers à Tellermann, en l'occurrence consoler, dialoguer, réparer, renommer le monde pour donner voix aux disparus, s'acheminer auprès d'eux au moyen de présences que cristallise le poème. Il s'agira d'ouvrir à de nouveaux possibles par l'expérience sensible qu'est le vers ou le mot polysémique. Nous observerons combien Tellermann reste perméable à l'Autre, particulièrement lorsque des dispositifs celaniens la relient au cosmos, l'enracinent dans un ordre symbolique et l'aident à prendre en compte à la fois l'Histoire et la musique de nos horizons intérieurs.

Dans le chapitre 5 , nous enrichirons cette analyse de dialectiques celaniennes. Sous votre nom se distingue par la profondeur de son dialogue avec Celan. Nous évaluerons ce dialogue comme manière de réinventer des mythes et des fables, de changer un passé douloureux en mouvement collectif vers un avenir plus apaisé. Trois axes d'étude - inventer, naviguer et ouvrir - montreront combien le fait de creuser la langue mène à des rencontres avec l'Autre. En effet, les rencontres poétiques entre Tellermann et Celan sont d'une richesse inouïe. En reprenant ses mots, rythmes et images, puis en en trouvant d'autres qui y répondent, Tellermann évoque le vide laissé par la Shoah tout en réécrivant différemment ce vide, en indiquant des facettes prometteuses qu'inscrivent les mots-cristaux celaniens. En tant que femme poète pleurant un bien-aimé, elle renverse d'ailleurs le mythe d'Orphée, dans la mesure où Celan est son Eurydice, sa «s sœur » $\left(S N 5^{\circ}\right)$ métaphorique. Le dialogisme servira à

20 Voir aussi Michael Bishop, « Esther Tellermann : exactitude, interrogation, psaume », Dystopie et poïein, agnose et reconnaissance : seize études sur la poésie française et francophone contemporaine, Amsterdam : Rodopi, 2014, p. 27-36. 
accueillir la mort, même la plus troublante ou indicible, comme autre face d'un «monde double » particulièrement intersubjectif. Un livre de John E. Jackson, Paul Celan : contre-parole et absolu poétique (2014), sera le point de départ pour de nombreuses réflexions.

Le chapitre 6 réfléchira au dialogisme à partir de Celan, Mandelstam et Nerval. Regarder en détail Le Troisième et Éternité à coudre à travers cette grille analytique nous permettra de comprendre les perspectives ouvertes sur le temps et l'espace, sur les liens entre soi et le monde que créent les motssymboles dans l'univers tellermannien et sur les chemins de la judéité qui s'ouvrent lorsque la venue en présence des mots instaure un acheminement vers le savoir, un accueil du devenir que nous apporte amoureusement la parole. Nous nous familiariserons avec « le Troisième » en tant que souhait d'un espace-temps lumineux, d'une union symbolique avec le cosmos à travers l'Autre. Nous écouterons, à l'instar de Mandelstam, un vibrato douloureusement muet, un lent jaillissement de motifs sur les chemins d'un inconscient troublé, inquiet. Nous saisirons divers sens de la notion de « coudre » l'éternité, par exemple l'idée de faire face aux larmes et aux blessures en tendant la main au langage et à l'Autre, et ce, afin de ressentir par le rite du poème un éventail de liens profonds aux plans affectif et ontologique. Nous suivrons une relation épistolaire destinée à faire apparaître ce «Elle » $(E C 8,28,29,36)$, la Poésie qui témoigne, et qui persévère dans ce témoignage malgré le temps qui passe, ses pas sur des planches étroites « épuisant le cour » $\left(E C{ }_{3} 6\right)$, mais nous rappelant la qualité charnelle et intersubjective du dire poétique. De nombreux gestes vers le sacré, y compris ceux dont l'empreinte se laisse apercevoir dans le tissu allusif, serviront de contrepoids à une douleur tant personnelle que collective. Plus on s'achemine à côté de Tellermann, plus le deuil, le désir et le langage se verront sous un jour nouveau.

Dans le chapitre 7, nous appliquerons une autre sorte de grille de lecture à l'œuvre tellermannienne, en nous appuyant sur un article de Michel Collot, « Reverdy selon Du Bouchet $» .{ }^{21}$ Nos remarques sur Avant la règle, Carnets à bruire et Un versant l'autre - ainsi que brièvement sur Un point fixe - s'inspireront de Carnets à bruire, hommage à André du Bouchet composé à partir de quelques-uns de ses carnets. Nous établirons des parallèles entre Reverdy, Du Bouchet et Tellermann. Nous considérerons des liens de parenté entre ces écrivains en mettant l'accent sur la manière dont on avance poétiquement dans le monde pour mieux être, par le regard et par les mots simples qui donnent à voir. En outre, nous aurons l'occasion de consulter quelques œuvres critiques

21 Michel Collot, « Reverdy selon Du Bouchet », Littérature 183 (2016/3), p. 94-106, <cairn. info>. 
de Reverdy et de Du Bouchet, assez révélatrices en ce qui concerne des procédés et perspectives tellermanniens.

Avec le chapitre 8, la boucle sera bouclée. Nous étudierons à travers Unversant l'autre le retour au calme, la légèreté retrouvée, le ton relativement adouci. Le noyau dur en sera l'image des « deux / oiseaux-voyageurs » (UV 87), synthèse des personnages ambigus que figure le récit tellermannien, de tous ceux qui parcourent le vivant. Nous tiendrons compte de la façon dont l'identité du lecteur évolue au fur et à mesure du contact avec ce récit, ces prières, ces versants du réel et du rêve qui donnent au réel son épaisseur. Là où le poète tend la main à l'Autre, ce sera dans une optique intersubjective assez optimiste, dans le souhait de voir chacun trouver son méridien, se lier à la langue et à la polyphonie que permettent les intertextes, sans oublier ni le vide ni le rien. Il sera question de l'adresse allusive à Celan ou à des proches, mais également d'autres actes de parole : voyager dans l'espace et le temps; découvrir l'intime de soi et du cosmos; creuser l'inconscient à travers la reprise cyclique de motifs ; faire avancer posément des séquences où s'entend la voix d'autrui, où se perçoivent des silhouettes « ruissel[antes] » (UV 152). Nous ferons état des alliances inépuisables qui en résultent, de futurs possibles partagés. En examinant divers sens du mot clé " coudre », nous noterons aussi les rapports singulièrement charnels et fleurissants avec le langage que mettent en place la poét(h)ique tellermannienne dans les recueils les plus récents. 